\title{
Central review of bone marrow biopsy specimens from patients with neuroblastoma
}

\author{
M M Reid, Borghild Roald for the European Neuroblastoma Study Group
}

\begin{abstract}
In order to achieve some uniformity in histological detection of bone marrow infiltration by neuroblastoma and to provide a measure of variation in histological opinions, sections from 712 evaluable trephine biopsy cores from children in a European Neuroblastoma Study Group (ENSG) study were reviewed centrally. Biopsy specimens were graded as tumour positive or negative. Discordance between local and central review opinions was found in 5\% of specimens. Only five of 165 children at presentation and nine of 256 re-staging procedures in 126 children, affecting one child each, had their diagnosis upgraded to positive. In six re-staging procedures, affecting one child each, the diagnosis was downgraded. The low discordance rate is encouraging and substantially less important than previously documented difficulties in obtaining adequate specimens.

(F Clin Pathol 1996;49:691-692)
\end{abstract}

Keywords: bone marrow trephine biopsy, children, neuroblastoma.

Bone marrow trephine biopsy, in addition to aspiration, is frequently part of the assessment of children with neuroblastoma. No centrally reviewed study of histological diagnoses from such biopsy specimens has been published. We report a large series of bone marrow biopsy specimens from children with neuroblastoma in which sections from all cores have been reviewed centrally. The purpose of the review process was to achieve some uniformity of histological opinion in children entered into European Neuroblastoma Study Group (ENSG) therapeutic trials, but it also provided the first opportunity to obtain some measure of the extent of variation in those opinions in a large series.

Department of

Haematology, Royal

Victoria Infirmary,

Queen Victoria Road,

Newcastle upon Tyne

NE1 4LP

M M Reid

Department of

Pathology, Ullevål

Hospital, Oslo, Norway

B Roald

Correspondence to:

Dr M M Reid.

Accepted for publication

4 June 1996

\section{Methods}

The study ran from January 1990 to July 1995. Sections of bone marrow biopsy specimens stained with haematoxylin and eosin or Giemsa (and often with a silver stain for reticulin) were examined by both authors. Ninety five per cent of the sections were the original slides that had been examined locally. The remainder were freshly stained sections from the original blocks. Immunohistochemical stains were not reviewed because few centres used them regularly, there is no uniformity in antibodies and their value remains in doubt. ${ }^{1}$ Evaluable biopsy specimens were graded as positive if frankly malignant tumour cells or ganglion-like neural tissue were present. All other evaluable biopsy specimens were graded as negative, irrespective of other possible abnormalities of cellularity, architecture, fibrosis or unidentifiable mononuclear cells. Evaluability has been discussed previously ${ }^{2}$ but, briefly, evaluable cores had to contain $0.5 \mathrm{~cm}$ or more of well preserved bone marrow after fixation unless definite tumour cells were present. Numbers of positive and negative cores were determined and for each core the reviewers' opinion was compared with that of the local pathologist in order that discordance rates could be calculated. For convenience, discordant results were described as false positive or false negative with no intention to beg the question about the right answer. Therefore, no formal calculation of sensitivity or specificity of local pathologists' reports was made. For each discordant result the impact of the change on the final histological diagnosis of the staging or re-staging procedure was determined. Results were grouped into those from presentation and those after treatment had started. Many children had more than one biopsy specimen taken during each staging or re-staging procedure, and many were re-staged more than once.

\section{Results}

Sections from 712 evaluable biopsy cores from 26 participating centres were graded. Table 1 summarises the results. As expected, most cores at presentation contained tumour while most after treatment had started did not. The discordance rate in both groups is approximately $5 \%$.

In only five of 165 children at presentation was tumour missed by the local pathologist and the histological diagnosis of the staging procedure upgraded to positive. The other eight false negative biopsy specimens were accompanied by another from the same child with locally detected tumour and therefore did not affect the staging. The single false positive biopsy specimen at presentation was from a child with an accompanying, infiltrated biopsy. Changing the result to negative did not affect the staging. Of 256 post-treatment, re-staging procedures the final histological diagnosis was upgraded in nine, and downgraded in six. In each case a different child was affected. The remaining five discordant, post-treatment results did not affect the staging. The reviewers thus disagreed with the local histological opinion in only $3 \%$ of presentation and $6 \%$ of post- 
Table 1 Summary of reviewers' opinions on bone marrow histology

\begin{tabular}{|c|c|c|c|c|c|c|c|}
\hline & \multicolumn{2}{|c|}{ Number of stagings } & \multicolumn{2}{|c|}{ Evaluable biopsy specimens } & \multicolumn{3}{|c|}{ Discordant biopsy specimens } \\
\hline & Total & Positive & Total & Positive & False positive & False negative & Total \\
\hline $\begin{array}{l}\text { Presentation }{ }^{\star} \\
\text { After treatment } \dagger\end{array}$ & $\begin{array}{l}165 \\
256\end{array}$ & $\begin{array}{l}129 \\
105\end{array}$ & $\begin{array}{l}279 \\
433\end{array}$ & $\begin{array}{l}208 \\
151\end{array}$ & $\begin{array}{l}1 \\
8\end{array}$ & $\begin{array}{l}13 \\
12\end{array}$ & $\begin{array}{l}14(5 \%) \ddagger \\
20(5 \%) \ddagger\end{array}$ \\
\hline
\end{tabular}

*At presentation, 165 children had one staging procedure each

†After treatment started, 126 children had one or more re-staging procedures.

$\neq$ Discordant results expressed as per cent of total evaluable biopsy specimens.

treatment staging procedures. Annual discordance rates over the five complete years of the study were $9 \%, 3 \%, 6 \%, 5 \%$, and $3 \%$. There were too few discordant results to allow meaningful centre by centre analysis and the 11 centres with no discordance submitted a mean of only four evaluable cores each.

\section{Discussion}

Current recommendations ${ }^{3}$ for staging and evaluating response to treatment in neuroblastoma include the use of trephine biopsy specimens as well as aspirates. This advice is based on the commonly experienced difficulty in detecting non-haemopoietic tumour metastases in aspirated bone marrow. Neuroblastoma is no exception. ${ }^{45}$

Despite the fact that most children with neuroblastoma are treated in specialist centres, few centres see large numbers of cases. Most pathologists thus have limited experience of detecting this tumour in bone marrow biopsy specimens. If routine histological examination of bone marrow biopsy specimens is important in evaluating the results of a therapeutic trial or in the management of individual patients, it should be of interest to establish the degree of variation in interpretation of this investigation. We have already documented notable variations in the adequacy of the core itself. ${ }^{2}$ This report concentrates on the histological opinions.

In most cases interpretation was straightforward. Discordance between the reviewers and local reporters usually involved small deposits of what, in the reviewers' opinion, was tumour that had been missed locally. There is no gold standard and the right answer is by no means clear in every case. There is a trend towards over diagnosing infiltration in the posttreatment biopsy specimens. In some cases we suspect that osteoblastic proliferation was misinterpreted as neuroblastoma but there were too few false positive cases to be certain. Discordance rates were reassuringly low $(5 \%)$ and affected the final staging procedure in only $3 \%$ at presentation and $6 \%$ post-treatment. There was no change with time. Discordance did not seem to affect some centres more than others, unlike the variations in adequacy of the biopsy core. ${ }^{2}$ Bone marrow biopsy is but one of many tests used to assess extent of disease and response to treatment. The whole weight of correctly staging/re-staging a patient does not rest on it alone. This low rate of discordance is therefore probably acceptable and unlikely to be improved on. In order to demonstrate convincingly a reduction in the discordance rate to $2.5 \%$ we would need to analyse at least 500 more staging/re-staging procedures.

Only crude, positive/negative assessments were analysed because, in effect, that is all that is required by current ENSG studies and internationally agreed criteria. ${ }^{3}$ Our study indicates that pathologists in the participating cen- $\vec{c}$ tres use similar, albeit sometimes ill defined 90 and subjective, criteria to detect tumour. Other 8 more subtle abnormalities such as abnormal architecture and pattern of fibrous tissue $e^{4}$ were $\stackrel{5}{5}$ also recorded by the reviewers. When the study is mature it may be possible to decide whether such abnormalities are clinically important. क् Should this be the case some reappraisal of the purpose of and need for central review may be necessary. Currently, the inadequate biopsy rate (average $17 \%$ ) and its variation between centres $^{2}$ is a substantially larger problem than differences in histological opinions about the presence or absence of tumour in the specimens.

We thank the United Kingdom Children's Cancer Study Group for organising and executing the transfer of the sections.

Participating centres: University Hospital of Aarhus; Royal Aberdeen Children's Hospital; Royal Belfast Hospital for Sick Aberdeen Children's H: Uspital; Royal Befast Rospital for Sick ' Children, Children's Hospital, Birmingham; Royal Hospital for Sick Children, Bristol; Addenbrooke's Hospital, Cambridge; Llandough Hospital, Cardiff; University Hospital, Copenhagen; Our Lady's Hospital for Sick Children, Dublin; Royal Hospital for Sick Children, Edinburgh; Royal Hospital for Sick Children, Glasgow; St James's University Hospital, Leeds; Leicester Royal Infirmary; Gasthuisberg, Leuven; St Bartholomew's Hospital, London; Hospital for Sick Children, London; Alder Hey Hospital, Liverpool; Royal Manchester Children's Hospital; I Royal Victoria Infirmary, Newcastle; Queen's University MediRoyal Victoria Inire (n) Radcliffe Hospital, Oxford; Derriford Hospital, Plymouth; Children's N Hospital, Sheffield; Southampton General Hospital; Royal o Marsden Hospital, Sutton.

1 Reid MM, Wallis JP, McGuckin AG, Pearson ADJ, Malcolm AJ. Routine histological compared with immunohistological examination of bone marrow trephine biopsy specimens $c$ in disseminated neuroblastoma. F Clin Pathol 1991;44:483-

Reid MM, Roald B. Adequacy of bone marrow trephine? biopsy specimens in children. F Clin Pathol 1996;49:226biops specimens in children. $f$ Chin Pachol 1996;49:226-

Brodeur G, Pritchard J, Berthold F, Carlsen NLT, Castel V, Castleberry RP, et al. Revisions of the international criteria for neuroblastoma diagnosis, staging and response to $\mathbb{D}$ treatment. F Clin Oncol 1993;11:1466-77.

4 Reid MM, Hamilton PJ. Histology of neuroblastoma involving the bone marrow: the problem of detecting residual tumour after initiation of treatment. $\mathrm{Br} \mathcal{f}$ Haematol 1988;69:487-90.

5 Carey PJ, Thomas L, Buckle G, Reid MM. Immunocytochemical examination of bone marrow in disseminated neuroblastoma. F Clin Pathol 1990;43:9-12.

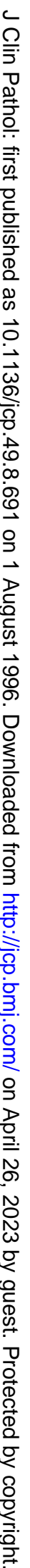

